\title{
Advances in Diagnostic Adjuncts for Oral Squamous Cell Carcinoma
}

\author{
Yi-Shing Lisa Cheng ${ }^{*}$ and John Wright
}

Diagnostic Sciences, Texas A\&M University Health Science Center-Baylor College of Dentistry, Dallas, Texas, USA

\begin{abstract}
Oral cancer is the $8^{\text {th }}$ most common cancer in males and the $15^{\text {th }}$ most common in females in the United States. Each year, it affects approximately 22,000 Americans and results in approximately 5300 deaths. The five-year survival rate of oral cancer remains low (53\% to $60 \%)$ for the past three decades and delayed diagnosis has been suggested to be one of the major reasons. The detection and diagnosis of oral cancer is currently based on clinical visual examination and histopathological evaluation of the biopsy material. In responding to the need for early detection of oral cancer, several diagnostic adjuncts have been developed over the years. The purpose of this article is to review the current knowledge about the commercially available diagnostic adjuncts as well as to review the research on the development of the promising tools for the early detection of oral cancer.
\end{abstract}

Keywords: Oral cancer, diagnostic adjuncts, salivary diagnostics.

\section{INTRODUCTION}

Oral cancer is the $11^{\text {th }}$ most common cancer in the world [1]. Each year approximately 22,000 Americans are diagnosed with it and about 5300 die. More than $90 \%$ of the oral cancers are squamous cell carcinoma (OSCC), which arises from the epithelial lining of the oral cavity. For the past three decades, the five-year survival rate of oral cancer has been improved some but remains in the range of $53 \%$ to $60 \%$, which is one of the lowest five-year survival rates of all the major cancer types [1,2]. Despite the fact that oral cavity is easily accessible for direct visual examination, most OSCC is not diagnosed until an advanced stage, which has been suggested to be one of the major reasons for a minimally improved survival rate [3]. This fact underscores the importance of patient education and the need to improve clinicians' ability to recognize early malignant and premalignant changes in the oral cavity.

In responding to the need for improving the clinicians' ability in detecting early malignant changes, various diagnostic adjuncts have been developed over the past few decades. The currently available diagnostic adjuncts for OSCC can be classified into the following three major categories: 1) Vital tissue staining (e.g. toludine blue or tulonium chloride), 2) Brush cytology (e.g., Oral CDx; from CDx Laboratories in Suffern, New York); and 3) Visualization adjunct (e.g., the ViziLite system, from Zila, Inc. of Phoenix, Arizona; MicroLux DL, from AdDent Inc., Danbury, CT; VELscope, from LED Dental Inc., of Vancouver, B.C. in Canada and Identafi 3000, from Trimima Remicalm of Houston, Texas) [4,5]. Some claimed to assist the differentiation between clinically benign and pre-malignant or malignant lesions (Oral CDx). Others aimed to detect pre-malignant or malignant changes that were not well recognized by clinical visual examination (toludine blue, ViziLite, MicroLux DL, VELscope and Indentafi 3000).

\footnotetext{
*Address correspondence to this author at the Diagnostic Sciences, TAMHSC-Baylor College of Dentistry, 3302 Gaston Avenue, Dallas, TX 75246, USA; Tel: 214-8288912; Fax: 214-8288306;
}

E-mail: ycheng@bcd.tamhsc.edu
Although many studies have investigated sensitivity and specificity of these diagnostic adjuncts, a survey and a recent critical evaluation of the published studies both concluded that there is still no convincing or adequate data to support their efficacy specifically for early detection or for reducing the death rate from OSCC [3-6]. Readers are strongly encouraged to read the recently published excellent reviews about these diagnostic adjuncts [3-5]. This mini review does not intend to repeat the content of the recent reviews on this subject and therefore, will only briefly discuss these currently available diagnostic adjuncts but extend the scope to the promising diagnostic adjuncts that are currently under development for early detection of oral cancer.

\section{CURRENTLY AVAILABLE DIAGNOSTIC ADJUNCTS}

\section{Vital Tissue Staining - Toludine Blue Staining}

Toludine blue is a metachromatic dye that binds to DNA in vital tissues. It is not cancer specific, but it has been reported to stain cells with relatively increased amounts of DNA and possibly altered DNA in premalignant and malignant epithelial lesions [4]. Toludine blue has been used for decades for screening abnormal epithelium in the cervix. Although it has not been approved by the FDA for use in the US as an oral cancer screening technique, it has been used as such for decades in other parts of the world [3].

Many studies have investigated sensitivity and specificity of toludine blue for detecting pre-malignant and malignant lesions, however, the results varied and were inconsistent [710]. Toludine blue staining appears to be useful as an adjunct to clinical examination in most studies, with a sensitivity ranging from $77-100 \%$ and a specificity ranging from 62 $100 \%[4,7,8,10]$. However, a low positive predictive value of $43.5 \%$ for potentially malignant lesions [10] and a falsenegative rate as high as $20.5 \%$ for pre-malignant lesions [8] also have been reported.

\section{Brush Cytology}

Brush cytology (Oral CDX, Fig. 1), developed in 1999, has become popular in dental offices in recent years [11-14]. 
This technique requires the clinician to collect epithelial cells by rotating a provided circular brush against the lesion until pinpoint bleeding is seen clinically, indicating penetration of the basement membrane and a collection of cells from all layers of the epithelium [11]. The cells are then fixed and sent to the company for a computer-assisted specimen analysis. The results are interpreted as falling into one of four categories: inadequate, negative, atypical, or positive; and the company recommends a follow-up scalpel biopsy for patients with an atypical or a positive result.

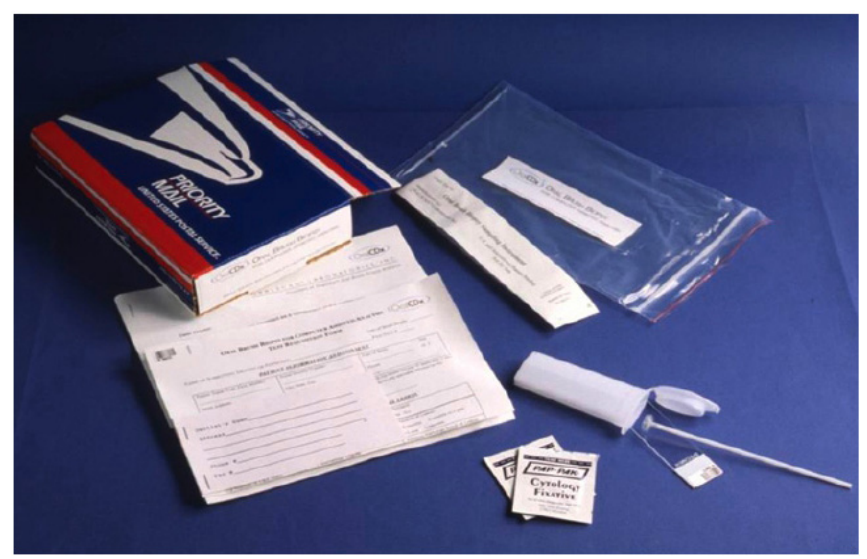

Fig. (1). The kit for Oral CDx from CDx Laboratories in Suffern, New York. It contains a cytobrush, a blank microscopic slide, a plastic container, two packages of fixative, a plastic bag and a test requisition form.

In a few studies comparing results of brush cytology to scalpel biopsies, sensitivity for detecting oral epithelial dysplasia or OSCC was reported high; ranging from $71.4 \%$ to $92 \%$ [15-17], although cases with false negative results have also been reported $[15,18]$. However, the specificity was reported as low, around $32 \%[15,16]$; and a high false positive rate (meaning initial "atypical" results from brush cytology, followed by scalpel biopsy that revealed no dysplasia or malignancy) was reported as ranging from 43 to $89 \%[16,19]$. In addition, a significant weakness of CDX is its inability to detect non-dysplastic leukoplakias which have a malignant transformation rate of approximately $1 \%$ [20].

\section{Visualization Adjuncts}

\section{Adjunct based on tissue reflectance-ViziLite, ViziLite Plus, MicroLux}

This category includes ViziLite (Fig. 2) and ViziLite Plus with TBlue from Zila Pharmaceuticals, Phoenix, and MicroLux DL from AdDent Inc., Danbury, Connecticut.

The ViziLite system and MicroLux are derived from an adjunct for diagnosing cervical lesions (speculoscopy) [4]. These products have been approved by the FDA to enhance the identification of oral mucosal lesions. For both systems, the patient is asked to rinse with a $1 \%$ acetic acid solution and then the oral cavity is examined by the clinician under the illumination of an activated chemiluminescent light (ViziLite) or a battery-operated light source (MicroLux DL). The differentiation between normal and abnormal oral epithelium is based on color appearance under the blue-white light generated by those two light sources. The normal epithelium appears light bluish whereas the abnormal epithelium appears distinctly white (aceto-white). The
ViziLite Plus also contains a tolonium chloride solution (TBlue) for marking the aceto-white lesion for subsequent biopsy once the light source is removed.

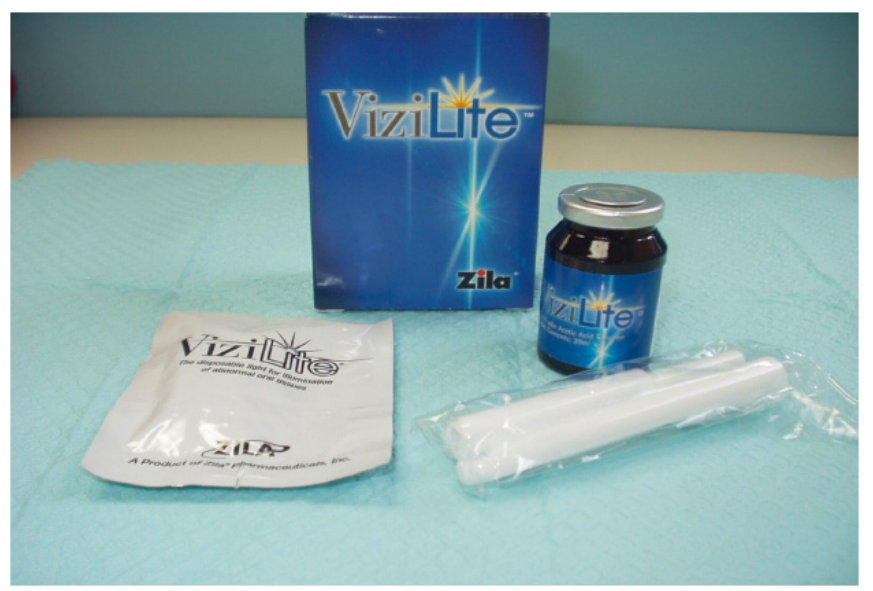

Fig. (2). The ViziLite System from Zila Pharmaceuticals, Phoenix. It consists of a brown bottle of $1 \%$ acetic acid solution and the disposable chemiluminescence light source package.

Several studies have investigated the benefit of using the ViziLite compared to conventional visual examination alone [21-26]. The ViziLite system was reported to highlight lesions of leukoplakia as well as oral benign lesions such as leukoedema, frictional keratosis, traumatic ulcer and lichen planusm [21, 23, 24]. Most of these studies either did not include comparison to the diagnostic gold standard i.e. histopathologic diagnosis based on a scalpel biopsy, or contained only a limited number of cases subsequently biopsied, which made it difficult to assess specificity and sensitivity $[21-24,27]$. There was only one study that correlated the ViziLite findings to the histopathologic diagnosis and the sensitivity, specificity and accuracy were reported to be $100 \%, 0 \%$ and $18.2 \%$, respectively [25]. Therefore, the system may provide little or very limited benefit over conventional visual examination in assessing clinically suspicious oral lesions $[3,25,26]$.

\section{Adjunct Based on Tissue Autofluorescence - VELscope}

The study of autofluorescence in normal and abnormal oral tissues has been an ongoing research interest in various regions of the world for the past four decades [28-34] and DeVeld et al. [35] provide an excellent review. Tissue autofluorescence is produced by fluorophores that exist in living tissue upon excitation with a suitable wavelength. The fluorophores can be in the extracellular matrix (collagen and elastin), in the blood (hemoglobin) or in the cells (keratin, the reduced form of nicotinamide adenine dinucleotide $(\mathrm{NADH})$ and flavin adenine dinucleotide (FAD)). The concentration of fluorophores changes in pathological conditions that alter the blood concentration, nuclear size distribution, collagen content and epithelial thickness [35]. Alterations in tissue autofluorescence have been observed in OSCC and in vivo autofluorescence imaging and spectroscopy have been applied for early detection of premalignant and malignant lesions in the oral cavity [3034].

The VELscope is a hand-held device that was approved by FDA for the direct visualization of autofluorescence in the oral cavity. It was recently introduced to the market as a 
diagnostic adjunct for oral cancer detection. The VELscope consists of a light source which emits an intense excitation blue light (400-460 nm) and a handpiece with a selective filter for direct visualization (Fig. 3) [3]. Under the illumination of this blue light, normal oral mucosa shows a pale green autofluorescence while abnormal tissue is proposed to show loss of autofluorescence and appears dark.

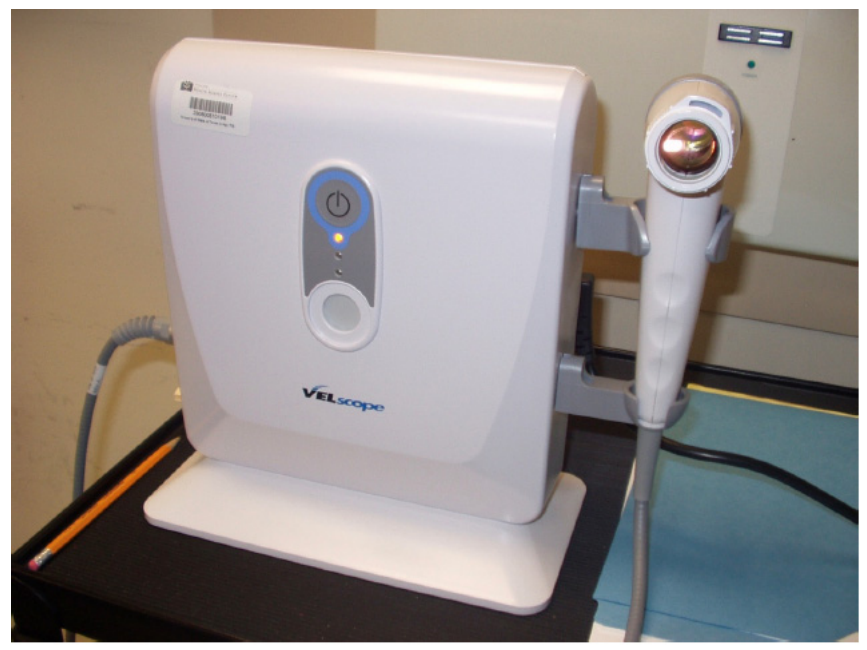

Fig. (3). The VELscope system from LED Dental Inc., White Rock, British Columbia, Canada. It consists of a light source which emits an intense excitation blue light (400-460 nm) and a handpiece with a selective filter for direct visualization.

The VELscope is a relative new device and so far only a limited number of studies have investigated its effectiveness as a diagnostic adjunct for oral cancer [36-39]. In the study reported by Lane et al. [36], clinical suspicious lesions (based on visual examination) in 44 patients were illuminated by VELscope followed by the biopsy. The sensitivity and specificity was reported to reach $98 \%$ and $100 \%$, respectively. Poh et al. [38] demonstrated the potential application of VELscope in the operating room in assisting the determination of the surgical margins for oral cancer. They examined the cancerous lesion with VELscope and recorded their finding before the patients underwent surgery. They found that the loss of autofluorescence extended beyond the clinically visible tumor margin in 19 of the 20 tumors and the extension varied from $4-25 \mathrm{~mm}$. Eighty-nine percent of these sampled areas of loss of autofluorescence showed the presence of carcinoma or epithelial dysplasia histologically. Poh et al. [37] also describe three cases where clinically normal mucosa showed loss of autofluorescence under VELscope examination. The biopsy of these three "lesions" revealed epithelial dysplasia or carcinoma-in-situ. This case report suggested the utility of VELscope in assisting clinical examination for screening purpose. Of note, these three studies all originated from British Columbia Cancer Agency [36-38]. Recently, Huber examined the oral cavity of 130 smokers with VELscope and compared the findings with conventional visual examination in an observational pilot study [39]. A total of 10 suspicious lesions were identified by conventional examination and no additional lesions were identified by VELscope. VELscope examination did not appear to enhance the detection of suspected lesions. In addition, Huber also noted that common oral inflammatory conditions, such as mucosal pigmentation, cheek biting, gingivitis, oral ulcerations and viral eruptions also frequently demonstrated loss of autofluorescence on VELscope examination. Therefore, the utilization of the VELscope as a general screening adjunct was also questioned by the author.

More studies are required in assessing the usefulness of VELscope for oral cancer screening.

\section{Adjunct Based on Tissue Autofluorescence and Reflectance-Identafi 3000}

Identafi 3000 , from Trimima ${ }^{\circledR}$ Remicalm of Houston, Texas, is the most recently developed device which was introduced to the market in early 2009. It uses white, violet and amber light to excite oral tissue and allows the clinicians to perform the conventional examination with the white light, to observe the changes in tissue autofluorescence with the violet light and to examine the areas of loss of autofluorrescence with the amber light [40]. The amber light illumination is proposed to enhance the reflectance property of normal tissue which will assist differentiation in the vasculature between normal and abnormal tissue. The vasculature in normal tissue is well defined in contrast to the abnormal tissue which was reported to show a diffuse vasculature. It is designed to identify lesions that may not be apparent to the naked eye. No research study has been reported to investigate the effectiveness of this new device yet.

\section{DEVELOPING DIAGNOSTIC ADJUNCTS}

\section{Salivary Diagnostics}

Salivary diagnostics, using saliva for disease diagnosis and health surveillance, for OSCC is a newly developing and ongoing area of research that shows considerable promise as a non-invasive method for early detection of oral cancer $[41,42]$. Several proteins, mRNAs, enzymes, and chemicals in the saliva are found sufficiently different in OSCC and normal controls to suggest that they might be potential biomarkers for OSCC. These potential salivary biomarkers for OSCC include 1) proteins of nuclear factor-kappa B (NF$\kappa \mathrm{B})$ dependent cytokines (tumor necrosis factor- $\alpha$ (TNF- $\alpha$ ), interleukin-1, interleukin-6, interleukin-8), basic fibroblast growth factor, Cyfra 21-1, cancer antigen- 125 (CA-125), tissue polypeptide antigen, endothelin; 2) mRNA of interleukin-8, interleukin-1, DUSP1 (dual specificity phosphatase 1), H3F3A (H3 histone family 3A), OAZ1 (ornithin decarboxylase antizyme 1), S100P (S100 calcium binding protein P), SAT (spermidine/spermine N1acetyltransferase EST); and 3) reactive nitrogen species (nitric oxide and nitrates), GST (glutathione S-transferase), SOD (superoxide dismutase) and 8-OHdG (8-hydroxydeoxyguanosine) [27, 43-48]. Recently, microRNAs (miRNA) also have been found in human saliva and two miRNAs, miR-125a and miR200a, were found to be present in significantly lower levels in the saliva of OSCC patients than in healthy controls [49]. This new finding makes miRNAs a new set of potential biomarkers for OSCC.

Salivary diagnostics has been one of the major funding focus for The National Institute of Dental and Craniofacial Research (NIDCR) for the past several years. In 2002, the NIDCR initiated a concerted research effort by giving seven U01 awards to develop microfluidics and microelectrico- 
mechanical systems (MEMS) for salivary diagnostics and now the progress is moving towards commercialization [50]. A handheld, automated device for rapid measuring multiple salivary biomarkers for oral cancer, the "Oral Fluidic NanoSensor Test", is currently under development by the University of California at Los Angels (UCLA) research team. It is estimated to be available for research and patient application in 3 years [50].

\section{In Vivo Confocal Microscopy}

Confocal microscopy is a well established imaging technique for research in cell biology. It provides the advantages of optical sectioning and high resolution imaging, achieved by blocking the light originating from tissue layers above and below the focal plane [51,52]. Confocal microscopy was originally used for study ex vivo specimens but has been applied to in vivo human tissues in recent years. In vivo confocal reflectance microscopy has demonstrated the ability to obtain images of tissue architecture and cell morphology with resolution similar to histology. In addition, because of the optical sectioning ability of the instrument, no surgical procedure, sectioning or staining would be required for this procedure. In vivo confocal images have been successfully obtained from the oral cavity [53-55]. A recent pilot study also showed that distinctive features such as nuclear irregularity and spacing in the images obtained by a fiber optic confocal reflectance microscope could be used to differentiate OSCC from normal oral mucosa [56]. Although further optimization of the instrument and validation of the data is still needed, the promising results provide preliminary optimism for another non-invasive tool for the early detection of oral cancer and premalignant lesions.

\section{CONCLUSION}

Several diagnostic adjuncts for detecting early changes for OSCC are currently available. Although many studies have investigated sensitivity and specificity of these diagnostic adjuncts, there is still no convincing or adequate data to support their efficacy specifically for early detection or for reducing the death rate from OSCC. A few new techniques, including salivary diagnostics and in vivo confocal microscopy, are in the development stage. Extensive research efforts have been devoted in discovering salivary biomarkers for the early detection of OSCC. An automated device for rapidly measuring multiple salivary biomarkers for oral cancer may be available in the near future.

\section{REFERENCE}

[1] Chi A. Squamous cell carcinoma. In: Neville BW, Damm DD, Allen CM, Bouquot JE, Eds. Oral and Maxillofacial Pathology. 3rd ed. St. Louis: Saunders Elsevier 2008: 409-21.

[2] Jemal A, Siegel R, Ward E, Hao Y, Xu J, Thun MJ. Cancer statistics, 2009. CA Cancer J Clin 2009; 59(4): 225-49.

[3] Lingen MW, Kalmar JR, Karrison T, Speight PM. Critical evaluation of diagnostic aids for the detection of oral cancer. Oral Oncol 2008; 44(1): 10-22.

[4] Kalmar JR. Advances in the detection and diagnosis of oral precancerous and cancerous lesions. Oral Maxillofacial Surg Clin N Am 2006; 18: 465-82.

[5] Patton LL, Epstein JB, Kerr AR. Adjunctive techniques for oral cancer examination and lesion diagnosis: a systematic review of the literature. J Am Dent Assoc 2008; 139(7): 896-905.
[6] Kujan O, Glenny AM, Oliver RJ, Thakker N, Sloan P. Screening programmes for the early detection and prevention of oral cancer. Cochrane Database Syst Rev 2006; 3: CD004150. Available from: http://onlinelibrary.wiley ${ }_{\text {ind }}$ /o/cochrane/clsysrev/articles/CD0041 50/frame.html [cited: $19^{\text {th }}$ Oct 2010].

[7] Rosenberg D, Cretin S. Use of meta-analysis to evaluate tolonium chloride in oral cancer screening. Oral Surg Oral Med Oral Pathol 1989; 67(5): 621-7.

[8] Warnakulasuriya KA, Johnson NW. Sensitivity and specificity of OraScan $(\mathrm{R})$ toluidine blue mouthrinse in the detection of oral cancer and precancer. J Oral Pathol Med 1996; 25(3): 97-103.

[9] Epstein JB, Oakley C, Millner A, Emerton S, Van der Meij E, Le $\mathrm{N}$. The utility of toluidine blue application as a diagnostic aid in patients previously treated for upper oropharyngeal carcinoma. Oral Surg Oral Med Oral Pathol Oral Radiol Endod 1997; 83(5): $537-47$.

[10] Onofre MA, Sposto MR, Navarro CM. Reliability of toluidine blue application in the detection of oral epithelial dysplasia and in situ and invasive squamous cell carcinomas. Oral Surg Oral Med Oral Pathol Oral Radiol Endod 2001; 91(5): 535-40.

[11] Sciubba JJ. Improving detection of precancerous and cancerous oral lesions. Computer-assisted analysis of the oral brush biopsy. U.S. Collaborative OralCDx Study Group. J Am Dent Assoc 1999; 130(10): 1445-57.

[12] Drinnan AJ. Screening for oral cancer and precancer--a valuable new technique. Gen Dent 2000; 48(6): 656-60.

[13] Svirsky JA, Burns JC, Carpenter WM, et al. Comparison of computer-assisted brush biopsy results with follow up scalpel biopsy and histology. Gen Dent 2002; 50(6): 500-3.

[14] Christian DC. Computer-assisted analysis of oral brush biopsies at an oral cancer screening program. J Am Dent Assoc 2002; 133(3): 357-62.

[15] Poate TW, Buchanan JA, Hodgson TA, et al. An audit of the efficacy of the oral brush biopsy technique in a specialist Oral Medicine unit. Oral Oncol 2004; 40(8): 829-34.

[16] Rick GM. Oral brush biopsy: The problem of false positives. Oral Surg Oral Med Oral Pathol Oral Radiol Endod 2003; 96(3): 252.

[17] Scheifele C, Schmidt-Westhausen AM, Dietrich T, Reichart PA The sensitivity and specificity of the OralCDx technique: Evaluation of 103 cases. Oral Oncol 2004; 40(8): 824-8.

[18] Potter TJ, Summerlin DJ, Campbell JH. Oral malignancies associated with negative transepithelial brush biopsy. J Oral Maxillofac Surg 2003; 61(6): 674-7.

[19] Slater LJ. Oral brush biopsy: false positives redux. Oral Surg Oral Med Oral Pathol Oral Radiol Endod 2004; 97(4): 419.

[20] Cowan CG, Gregg TA, Napier SS, McKenna SM, Kee F. Potentially malignant oral lesions in northern Ireland: a 20-year population-based perspective of malignant transformation. Oral Dis 2001; 7(1): 18-24.

[21] Huber MA, Bsoul SA, Terezhalmy GT. Acetic acid wash and chemiluminescent illumination as an adjunct to conventional oral soft tissue examination for the detection of dysplasia: a pilot study. Quintessence Int 2004; 35(5): 378-84.

[22] Ram S, Siar CH. Chemiluminescence as a diagnostic aid in the detection of oral cancer and potentially malignant epithelial lesions. Int J Oral Maxillofac Surg 2005; 34(5): 521-7.

[23] Epstein JB, Gorsky M, Lonky S, Silverman S, Jr., Epstein JD, Bride M. The efficacy of oral lumenoscopy (ViziLite) in visualizing oral mucosal lesions. Spec Care Dentist 2006; 26(4): 171-4.

[24] Kerr AR, Sirois DA, Epstein JB. Clinical evaluation of chemiluminescent lighting: an adjunct for oral mucosal examinations. J Clin Dent 2006; 17(3): 59-63.

[25] Farah CS, McCullough MJ. A pilot case control study on the efficacy of acetic acid wash and chemiluminescent illumination (ViziLite) in the visualisation of oral mucosal white lesions. Oral Oncol 2007; 43(8): 820-4.

[26] Oh ES, Laskin DM. Efficacy of the ViziLite system in the identification of oral lesions. J Oral Maxillofac Surg 2007; 65(3): 424-6.

[27] Bahar G, Feinmesser R, Shpitzer T, Popovtzer A, Nagler RM. Salivary analysis in oral cancer patients: dNA and protein oxidation, reactive nitrogen species, and antioxidant profile. Cancer 2007; 109(1): 54-9. 
[28] De Veld DC, Sterenborg HJ, Roodenburg JL, Witjes MJ. Effects of individual characteristics on healthy oral mucosa autofluorescence spectra. Oral Oncol 2004; 40(8): 815-23.

[29] De Veld DC, Skurichina M, Witjes MJ, et al. Autofluorescence characteristics of healthy oral mucosa at different anatomical sites. Lasers Surg Med 2003; 32(5): 367-76.

[30] Betz CS, Mehlmann M, Rick K, et al. Autofluorescence imaging and spectroscopy of normal and malignant mucosa in patients with head and neck cancer. Lasers Surg Med 1999; 25(4): 323-34.

[31] Chen CT, Chiang HK, Chow SN, et al. Autofluorescence in normal and malignant human oral tissues and in DMBA-induced hamster buccal pouch carcinogenesis. J Oral Pathol Med 1998; 27(10): 4704.

[32] Wang CY, Chiang HK, Chen CT, Chiang CP, Kuo YS, Chow SN. Diagnosis of oral cancer by light-induced autofluorescence spectroscopy using double excitation wavelengths. Oral Oncol 1999; 35(2): 144-50.

[33] Onizawa K, Okamura N, Saginoya H, Yoshida H. Characterization of autofluorescence in oral squamous cell carcinoma. Oral Oncol 2003; 39(2): 150-6.

[34] De Veld DC, Skurichina M, Witjes MJ, Duin RP, Sterenborg HJ, Roodenburg JL. Autofluorescence and diffuse reflectance spectroscopy for oral oncology. Lasers Surg Med 2005; 36(5): 35664.

[35] De Veld DC, Witjes MJ, Sterenborg HJ, Roodenburg JL. The status of in vivo autofluorescence spectroscopy and imaging for oral oncology. Oral Oncol 2005; 41(2): 117-31.

[36] Lane PM, Gilhuly T, Whitehead P, et al. Simple device for the direct visualization of oral-cavity tissue fluorescence. J Biomed Opt 2006; 11(2): 024006.

[37] Poh CF, Ng SP, Williams PM, et al. Direct fluorescence visualization of clinically occult high-risk oral premalignant disease using a simple hand-held device. Head Neck 2007; 29(1): 71-6.

[38] Poh CF, Zhang L, Anderson DW, et al. Fluorescence visualization detection of field alterations in tumor margins of oral cancer patients. Clin Cancer Res 2006; 12(22): 6716-22.

[39] Huber MA. Assessment of the VELscope as an adjunctive examination tool. Tex Dent J 2009; 126(6): 528-35.

[40] Trimira.net [homepage on the Internet]. Houston: Trimira ${ }^{\circledR}$ Remicalm; c 2010 [cited September 13, 2009]; Available from: http://trimira.net/identafi

[41] Wong DT. Salivary diagnostics for oral cancer. J Calif Dent Assoc 2006; 34(4): 303-8.

[42] Wong DT. Salivary diagnostics powered by nanotechnologies, proteomics and genomics. J Am Dent Assoc 2006; 137(3): 313-21.
[43] Li Y, St John MA, Zhou X, et al. Salivary transcriptome diagnostics for oral cancer detection. Clin Cancer Res 2004; 10(24): 8442-50.

[44] St John MA, Li Y, Zhou X, et al. Interleukin 6 and interleukin 8 as potential biomarkers for oral cavity and oropharyngeal squamous cell carcinoma. Arch Otolaryngol Head Neck Surg 2004; 130(8): 929-35.

[45] Rhodus NL, Ho V, Miller CS, Myers S, Ondrey F. NF-kappaB dependent cytokine levels in saliva of patients with oral preneoplastic lesions and oral squamous cell carcinoma. Cancer Detect Prev 2005; 29(1): 42-5.

[46] Nagler R, Bahar G, Shpitzer T, Feinmesser R. Concomitant analysis of salivary tumor markers-a new diagnostic tool for oral cancer. Clin Cancer Res 2006; 12(13): 3979-84

[47] Pickering V, Jordan RC, Schmidt BL. Elevated salivary endothelin levels in oral cancer patients-A pilot study. Oral Oncol 2007; 43(1): 37-41.

[48] Vucicevic Boras V, Cikes N, Lukac J, Virag M, Cekic-Arambasin A. Salivary and serum interleukin 6 and basic fibroblast growth factor levels in patients with oral squamous cell carcinoma. Minerva Stomatol 2005; 54(10): 569-73.

[49] Park NJ, Zhou H, Elashoff D, et al. Salivary microRNA: discovery, characterization, and clinical utility for oral cancer detection. Clin Cancer Res 2009; 15(17): 5473-7.

[50] Lee JM, Garon E, Wong DT. Salivary diagnostics. Orthod Craniofac Res 2009; 12(3): 206-11.

[51] Mongan LC, Gormally J, Hubbard AR, D'Lacey C, Ockleford CD. Confocal microscopy. Theory and applications. Methods Mol Biol 1999; 114: 51-74.

[52] Paddock SW. Confocal laser scanning microscopy. Biotechniques 1999; 27(5): 992-6.

[53] White WM, Rajadhyaksha M, Gonzalez S, Fabian RL, Anderson RR. Noninvasive imaging of human oral mucosa in vivo by confocal reflectance microscopy. Laryngoscope 1999; 109(10): 1709-17.

[54] Zheng W, Harris M, Kho KW, et al. Confocal endomicroscopic imaging of normal and neoplastic human tongue tissue using ALAinduced-PPIX fluorescence: a preliminary study. Oncol Rep 2004; 12(2): 397-401.

[55] Thong PS, Olivo $\mathrm{M}$, Kho $\mathrm{KW}$, et al. Laser confocal endomicroscopy as a novel technique for fluorescence diagnostic imaging of the oral cavity. J Biomed Opt 2007; 12(1): 014007.

[56] Maitland $\mathrm{KC}$, Gillenwater AM, Williams $\mathrm{MD}$, et al. In vivo imaging of oral neoplasia using a miniaturized fiber optic confocal reflectance microscope. Oral Oncol 2008; 44(11): 1059-66.

This is an open access article licensed under the terms of the Creative Commons Attribution Non-Commercial License (http://creativecommons.org/licenses/by-nc/ 3.0/) which permits unrestricted, non-commercial use, distribution and reproduction in any medium, provided the work is properly cited. 